\title{
A Case of a Cavitary Iris Melanoma with Aqueous Cytological Modulation
}

\author{
Lindsay A. McGrath ${ }^{a}$ James F. Bacon ${ }^{b}$ Paul Rundle ${ }^{a}$ Ian G. Rennie ${ }^{a}$ \\ Hardeep Singh Mudhar ${ }^{\mathrm{c}}$ \\ ${ }^{a}$ Sheffield Ocular Oncology Service, Royal Hallamshire Hospital, Sheffield, UK; ${ }^{\text {b}}$ Eye Institute, Cleveland Clinic Abu \\ Dhabi, Abu Dhabi, UAE; ' National Specialist Ophthalmic Pathology Service (NSOPS), Department of Histopathology, \\ Royal Hallamshire Hospital, Sheffield, UK
}

\section{Established Facts}

- Cavitations can occur in uveal melanomas, but have been reported in less than $1 \%$ of cases.

- When uveal melanoma is exposed to aqueous humour, it can undergo cytological modification with downgrading of atypia.

\section{Novel Insights}

- Cavitations can also be seen in primary melanoma of the iris.

- Care should be taken in the interpretation of a suspected iris tumour biopsy, as melanomas in this region may undergo cytological modification.

\section{Keywords}

Iris melanoma $\cdot$ Uveal melanoma · Iris cyst

\begin{abstract}
Malignant melanoma is the most common primary malignant tumour of the iris, but represents a small proportion of all uveal melanomas. The authors describe a 34-year-old male with a pigmented lesion of the iris. The lesion remained stable for 7 years, but the patient re-presented after this time with sudden enlargement of the mass and hyphaema. Excisional biopsy confirmed cavitary melanoma of the iris. This is the first reported case of cavitation in a primary iris melanoma. The patient has not had any further adjuvant treatment and remains metastasis free at 5 years of follow-up.
\end{abstract}

(c) 2018 S. Karger AG, Basel

\section{KARGER}

(๑) 2018 S. Karger AG, Basel

E-Mail karger@karger.com

www.karger.com/oop
Iris cysts can present diagnostic and therapeutic challenges, particularly in their differentiation from iris and ciliary body melanoma. Although advancement in imaging modalities allows more detailed analysis of intraocular lesions, they can occasionally present with atypical features causing diagnostic dilemma. Cavitation is known to occur in less than $1 \%$ of intraocular melanomas and is most common in the ciliary body $[1,2]$. The ultrasound findings can be difficult to differentiate from those of a benign epithelial cyst.

We describe a 34-year-old male with a long-standing cystic pigmented lesion of the iris, which evolved to a cavitary melanoma. 

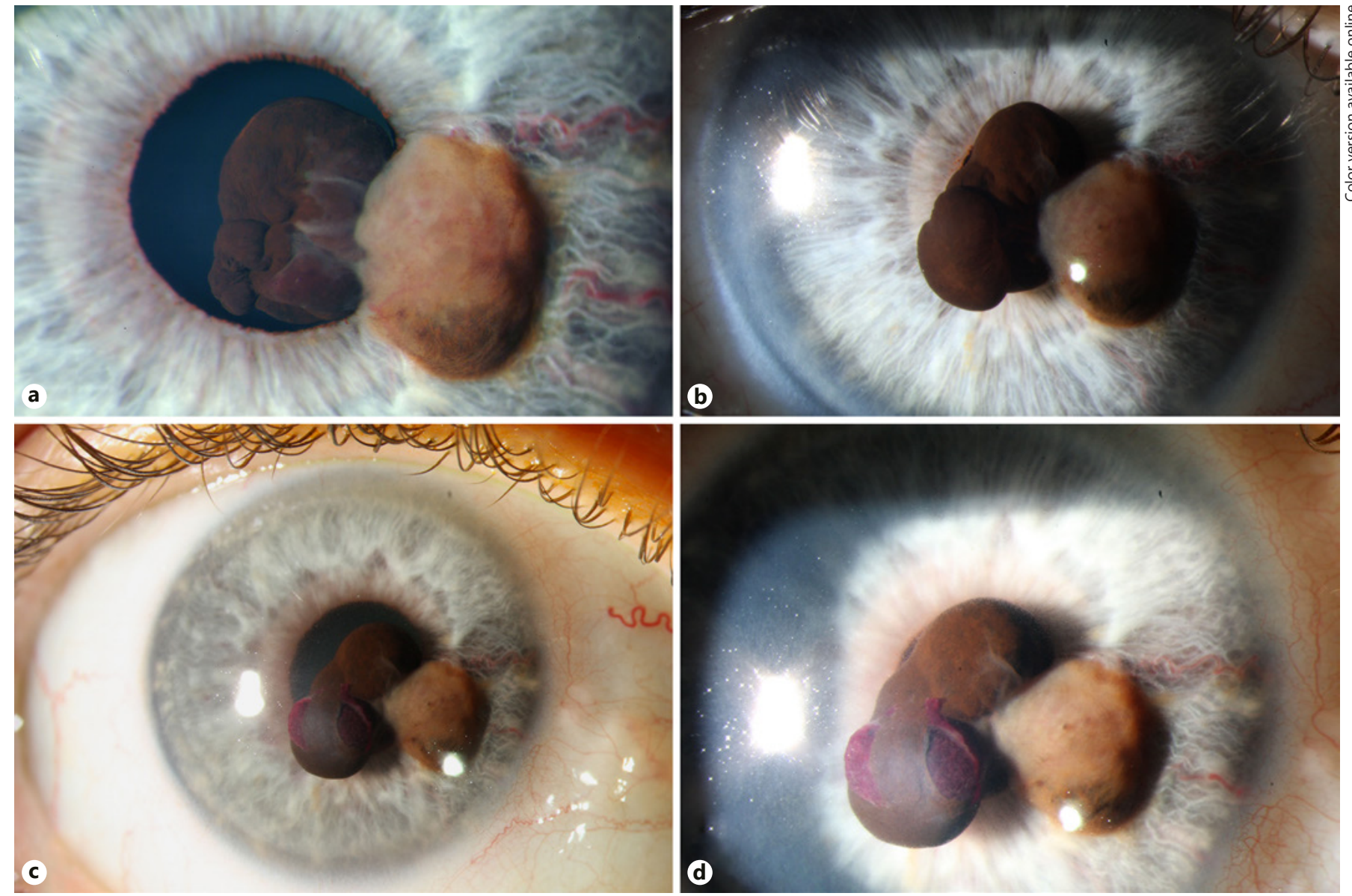

Fig. 1. a Right pupillary margin lesion at presentation: a 3-mm dumbbell-shaped mass covered with pigmented epithelium from the back surface of the iris encroaching on the pupil and a $3 \times 2.5$ $\mathrm{mm}$ partially melanotic lesion entering the anterior chamber from the front of the iris. This lesion had enlarged feeder vessels and a slight dusting of pigment spread $1 \mathrm{~mm}$ from the lesion but not

reaching the edge of the iris or angle on gonioscopy. b Four years after presentation, the lesion was noted to have slow growth, gradually encroaching the visual axis, although vision remained stable. c, d A haemorrhagic outgrowth from the inferotemporal edge of the retroiridal portion of the lesion.

\section{Case Report}

A 34-year-old male was referred with a pigmented thickening of the right inferonasal iris (Fig. 1a). Ultrasound biomicroscopy revealed an acoustically solid lesion measuring $5.86 \times 6.42 \times 2.91 \mathrm{~mm}$ with multiple well-defined echo-lucent cavities, thought to represent benign pigment epithelial cysts (Fig. 2a). The patient had a pigmented area of the iris since puberty, but had recently noticed photophobia and blurred vision. The lesion had variegated pigmentation and suspicious afferent blood vessels, and therefore the patient underwent aspiration biopsy under local anaesthetic. Cytological examination concluded the lesion to be a spindle cell naevus of the iris. The patient was followed 6-monthly with minimal change to his anterior segment (Fig. 1b), and vision was preserved at 6/6.

The patient re-presented 7 years after initial presentation with sudden onset blurred vision in the right eye and throbbing headache. On examination, the vision was reduced to hand movements

in the affected eye. Intraocular pressure in the right eye was $47 \mathrm{~mm}$ $\mathrm{Hg}$, and slit-lamp examination revealed a micro-hyphaema which appeared to originate from the iris cyst (Fig. 1c, d). Ultrasound biomicroscopy showed enlargement of the solid portion of the iris mass, with persistent thick-walled cystic spaces posteriorly (Fig. 2b).

The patient underwent iridocyclectomy due to the acute symptoms and documented growth, concerning for malignancy. Histopathology showed an iris tumour comprising three zones. The first was a solid tumour zone (Fig. 3a, asterisk). The second zone was a cavitary area surrounded by solid tumour (Fig. 3a, larger black arrow, and Fig. 3b, asterisk). The third zone comprised an iris pigment epithelial cyst (Fig. 3a, smaller black arrow). At higher power, the solid zone comprised atypical melanocytes showing variable nuclear size and variable chromasia with some nuclei containing conspicuous nucleoli (Fig. 3c). These were the features of an iris melanoma. The melanoma cells lining the cavity were of an identical nature (Fig. 3d). The thickness of the melanoma cells lining the 
cavity and iris pigment epithelium varied from several layers thick (Fig. 3e) to only one to two cells thick (Fig. 3f) to no tumour cells, with just iris pigment epithelium (Fig. $3 \mathrm{~g}$ ).

In the solid melanoma zone, the anterior face exposed to aqueous showed cells with a smaller size, with smaller nuclei and less cytoplasm and resembling naevus type cells (Fig. 3h). When immunohistochemically stained with cyclin $\mathrm{D} 1$, the proportion of melanoma cells showing nuclear staining was much greater in the larger cells in the stroma (Fig. 3i), compared to the cells on the anterior face (Fig. 3j). The iris melanoma was staged as T1a (American Joint Cancer Classification scheme, 8th edition) and genetic testing showed no evidence of chromosome 3 or 8 changes by fluorescence in situ hybridisation [3].

The patient remained stable for 5 years postoperatively, with no recurrence of tumour or metastasis, and best corrected visual acuity of $6 / 6$ in the affected eye. The intraocular pressure remained within normal limits without ocular hypotensives.

\section{Discussion}

Cavitation within uveal melanomas is rare, with less than 30 reported cases in the English literature $[1,4]$. To the best of our knowledge, this is the first case of a cavitary melanoma of the iris to be described. Cavitation of an intraocular melanoma is rarely appreciated clinically, and when present may masquerade as a different entity, delaying definitive treatment of the disease.

Cavitations in melanomas are seldom apparent on slitlamp examination and usually require ultrasound for diagnosis. Ultrasonographically, cavitations present as single or multiple, hollow, cystic-like cavities surrounded by a thick reflective wall $[4,5]$. Uncommonly, they may be solid lesions with single or multiple acoustically hollow areas. Anterior segment optical coherence tomography can also be useful in providing high-resolution margins of iris tumours.

In a histopathologic study published in 1948, Kennedy [6] was the first to report a ciliary body melanoma with a large cavity. The favoured hypothesis for the development of cavitary changes in malignant melanomas is tumour necrosis and haemorrhage $[2,6,7]$. It is postulated that an imbalance between blood supply and tumour growth could lead to necrosis and tissue reabsorption [2]. Some authors speculate the accumulation of muco-proteinaceous substances or serous exudation may play a role [6-9].

Following excision, histopathology demonstrated a pigment epithelial cyst continuous with the main nodular iris melanoma in our case. The pigment epithelial cyst was lined by varying thicknesses of melanoma cells from several cells thick to only one to two cell layers thick, to no tumour cells. Previous reports of cavitary ciliary body melanomas failed to identify endothelial or epithelial cells
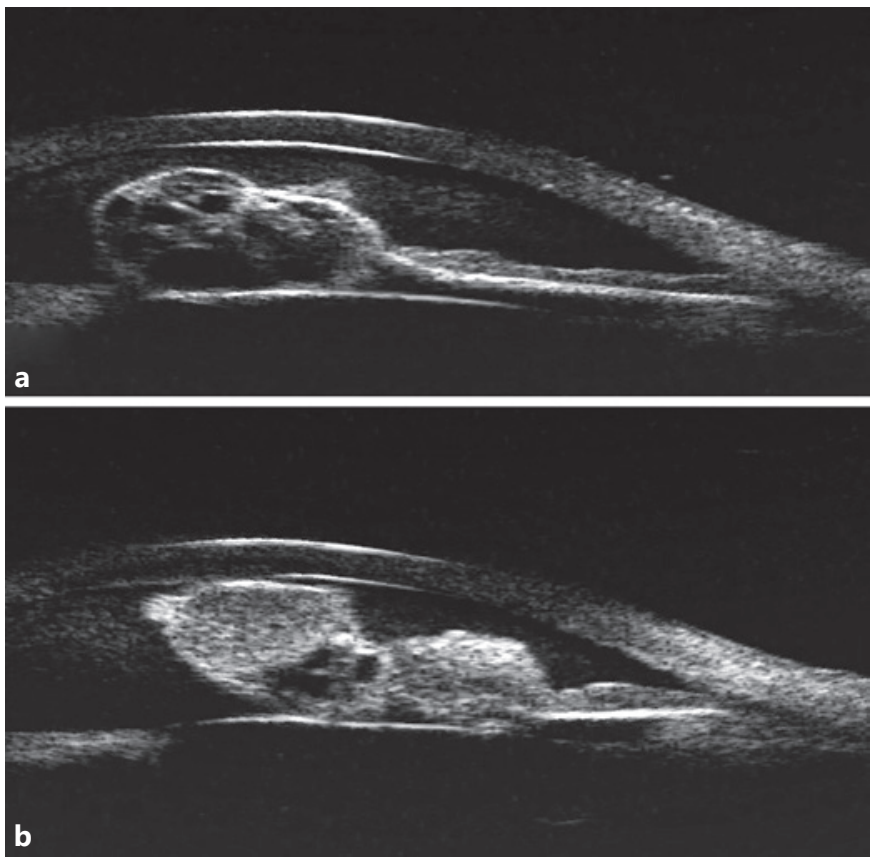

Fig. 2. Transverse ultrasound biomicrosopy images from 4:00 in the right eye. a At initial presentation, a solid mass arising from the iris stroma centrally with a small cavitation at the pupillary portion of the lesion can be seen. $\mathbf{b}$ After rupture of the cyst, prominence of the pupillary portion of the mass is visible, with larger lucencies involving the posterior iris stroma.

lining the inner walls of the cavities $[2,6,8,9]$. In contrast, primary central and midzonal iris cysts tend to be lined by pigment epithelium, while stromal cysts are lined with stratified squamous epithelium [10].

In the evaluation of a cystic lesion of the iris, differentiation of a thick-walled cavitation in melanoma from thin-walled benign iris pigment epithelial cyst, with little comparative information, can be very challenging for the clinician [4]. Iris pigment epithelial cysts typically arise near the iris root, in the iridociliary sulcus [11]. They are often smaller than cavitary melanomas and appear clear or finely dusted with surface pigment [2]. Typically, the reflective wall of an iris pigment epithelium cyst measures $100 \mu \mathrm{m}$ or less [12]. In contrast, ciliary body melanoma shows thicker-walled cavitary spaces of $200 \mu \mathrm{m}$ to several millimetres [2]. Other differential diagnoses to consider include iris stromal cyst, medulloepithelioma, and cystic adenoma of the pigmented ciliary epithelium. The diameter of the cysts can also be useful in differentiating between the latter, where true cysts in adenomas can measure up to $100 \mu \mathrm{m}$, those in cavitary melanoma typically start at $500 \mu \mathrm{m}$ in width [13]. 
Fig. 3. Histology. a Haematoxylin and eosin (H\&E)-stained section showing three zones. The asterisk represents the solid melanoma, the larger arrow shows the cavitary component, and the smaller arrow the pigment epithelial cyst component. b Higher power of a showing the cavity marked by the asterisk and melanoma constituting the walls of the cavity. $\mathrm{c} \mathrm{H} \& \mathrm{E}$-stained section of the cytological detail of the solid melanoma area. The nuclei show varying chromasia and size variation. Some contain nucleoli. Melanin pigment is present. $\mathbf{d} \mathrm{H} \& \mathrm{E}$ of the melanoma lining the cavity shows similar cytological features to c. e H\&E showing melanoma several cell layers thick lining the iris pigment epithelium. f H\&E showing melanoma cells up to 2 cells thick (arrow) lining the iris pigment epithelium. g H\&E showing just iris pigment epithelium without a tumour lining. $\mathbf{h}$ Melanoma cells on the anterior iris surface showing smaller cell size (upper plate) compared to deeper melanoma cells (lower plate). i, j Cyclin D1 immunohistochemistry showing many more nuclei positive in the deeper, larger melanoma cells, compared to the surface, smaller melanoma cells.
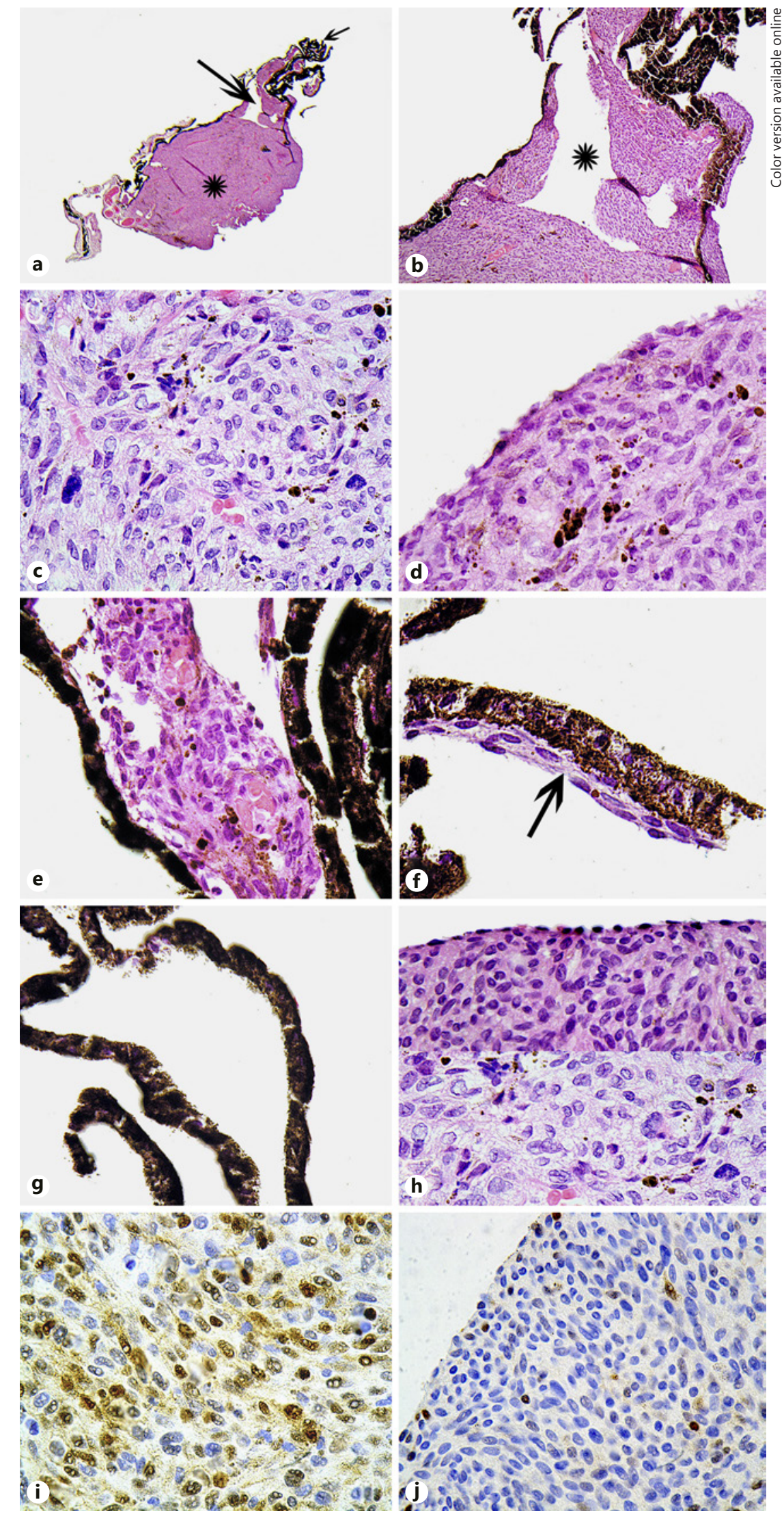
The initial cytology of the iris lesion revealed features of a benign spindle cell naevus. The subsequent diagnosis of melanoma questions if the original lesion underwent malignant transformation or the biopsy was taken from a benign part of the iris lesion (sampling error). However, it is well described that uveal melanoma that is exposed to aqueous humour can downgrade the cytological atypia with reduction in proliferation indices, closely mimicking melanocytic naevus [14]. We previously described this phenomenon with ciliary body melanoma invading the iris, representing a micro-environmental shift from melanoma not exposed to aqueous, to aqueous exposure. We showed that when ciliary body melanoma invaded the iris surface and was exposed to aqueous, it displayed reduction in tumour cell size with reduced expression of cyclin D1 (a marker of cell proliferation) compared to the deeper iris stromal and ciliary body melanoma cells. Furthermore, a significant number of cases of the modified melanoma cells showed fewer cytogenetic aberrations (chromosome 3 and 8 changes) when compared to the ciliary body and iris stromal melanoma components in the same paraffin sections. It is clear from this case report that the same observations hold true for primary iris melanoma exposed to aqueous. While the factors that may cause this remain speculative, a favourite candidate may be ascorbic acid. Ascorbic acid has 15 to 25 times the concentration in the anterior chamber compared to plasma in mammals $[15,16]$, with recent studies demonstrating its anti-tumour activity against melanoma in vitro [17].

Clinicians should be aware that iris melanomas can rarely contain cavitations, and be familiar with features which distinguish these lesions from other benign aetiologies. On ultrasound, cystic cavities may be isolated or numerous with thick walls of medium reflectivity.

\section{Statement of Ethics}

The study protocol was approved by the Royal Hallamshire Hospital committee on human research and the patient gave informed consent for photographs and clinical information to be published.

\section{Disclosure Statement}

There are no financial disclosures or conflicts of interest to disclose. All authors contributed to the development of the manuscript.

\section{References}

1 Criss JS, Shields CL, Materin MA, Reichel E, Eagle RC Jr, Shields JA. Progressive enlargement of cavity within melanoma masquerading as iris cyst [published Online First: 2006/10/28]. Cornea. 2006 Aug;25(7):863-5.

2 Lois N, Shields CL, Shields JA, Eagle RC Jr, De Potter P. Cavitary melanoma of the ciliary body. A study of eight cases [published Online First: 1998/06/17]. Ophthalmology. 1998 Jun;105(6):1091-8.

3 Kivelä T, Simpson ER, Grossniklaus HE, et al. Uveal melanoma. AJCC Cancer Staging Manual. 8th ed. New York: Springer; 2017. pp. 805-17.

4 Jang J, Kaliki S, Eagle RC, Shields JA, Shields CL. Multicavitary ciliary body melanoma presenting as a cyst [published Online First: 2013/06/19]. Oman J Ophthalmol. 2013 Jan; 6(1):48-50.

5 Becerra EM, Saornil MA, Blanco G, Méndez MC, Muiños Y, Esteban MR. Cavitary choroidal melanoma [published Online First: 2006/01/05]. Can J Ophthalmol. 2005 Oct; 40(5):619-22.

6 Kennedy RE. Cystic malignant melanomas of the uveal tract [published Online First: 1948/02/01]. Am J Ophthalmol. 1948 Feb; 31(2):159-67.
7 Zhang J, Demirci H, Shields CL, Leon JA, Shields JA, Eagle RC Jr. Cavitary melanoma of ciliary body simulating a cyst [published Online First: 2005/04/13]. Arch Ophthalmol. 2005 Apr;123(4):569-71.

8 Scott CT, Holland GN, Glasgow BJ. Cavitation in ciliary body melanoma [published Online First: 1997/02/01]. Am J Ophthalmol. $1997 \mathrm{Feb} ; 123(2): 269-71$.

9 Zakka KA, Foos RY, Spencer WH, Kerman BM, Newman NM, Pettit TH. Cavitation in intraocular malignant melanoma [published Online First: 1982/01/01]. Arch Ophthalmol. 1982 Jan;100(1):112-4.

10 Rao A, Gupta V, Bhadange Y, Sharma R, Shields JA. Iris cysts: a review [published Online First: 2011/02/01]. Semin Ophthalmol. 2011 Jan;26(1):11-22.

11 Augsburger JJ, Affel LL, Benarosh DA. Ultrasound biomicroscopy of cystic lesions of the iris and ciliary body [published Online First: 1996/01/01]. Trans Am Ophthalmol Soc. 1996;94:259-71.

12 Lois N, Shields CL, Shields JA, Mercado G. Primary cysts of the iris pigment epithelium. Clinical features and natural course in $234 \mathrm{pa}-$ tients [published Online First: 1998/10/27]. Ophthalmology. 1998 Oct;105(10):1879-85.
13 Shields JA, Shields CL. Clinical features of posterior uveal melanoma. In: Shields JA, Shields CL, editors. Intraocular Tumors: An Atlas and Textbook. Philadelphia: Lippincott Williams and Wilkins; 2008. pp. 86-94.

14 Mudhar HS, Saunders E, Rundle P, Rennie IG, Sisley K. The in vivo modulatory effects of an anterior-chamber microenvironment on uveal melanoma [published Online First: 2008/11/21]. Br J Ophthalmol. 2009 Apr; 93(4):535-40.

15 Reiss GR, Werness PG, Zollman PE, Brubaker RF. Ascorbic acid levels in the aqueous humor of nocturnal and diurnal mammals [published Online First: 1986/05/01]. Arch Ophthalmol. 1986 May;104(5):753-5.

16 Kronfeld PC. The ascorbic acid content of the aqueous of surgically aphakic human eyes [published Online First: 1952/01/01]. Trans Am Ophthalmol Soc. 1952;50:347-58.

17 Fischer AP, Miles SL. Ascorbic acid, but not dehydroascorbic acid increases intracellular vitamin $\mathrm{C}$ content to decrease Hypoxia Inducible Factor -1 alpha activity and reduce malignant potential in human melanoma [published Online First: 2016/12/26]. Biomed Pharmacother. 2017 Feb;86:502-13. 